\title{
ORKAMBI-Mediated Rescue of Mucociliary Clearance in Cystic Fibrosis Primary Respiratory Cultures Is Enhanced by Arginine Uptake, Arginase Inhibition, and Promotion of Nitric Oxide Signaling to the Cystic Fibrosis Transmembrane Conductance Regulator Channel[ $\$$
}

Yu-Sheng Wu, Janet Jiang, Saumel Ahmadi, Alexandria Lew, Onofrio Laselva, Sunny Xia, Claire Bartlett, Wan Ip, Leigh Wellhauser, Hong Ouyang, Tanja Gonska, Theo J. Moraes, and Christine E. Bear

Programmes in Translational Medicine (Y.-S.W., C.B., W.I., H.O., T.G., T.J.M.) and Molecular Medicine (Y.-S.W., J.J., S.A., A.L., O.L., S.X., L.W., C.E.B.), Research Institute, Hospital for Sick Children, Toronto, Ontario, Canada; and Departments of Laboratory Medicine and Pathobiology (T.J.M.), Biochemistry (C.E.B.), Physiology (Y.-S.W., S.A., O.L., S.X., C.E.B.), and Paediatrics (T.G., T.J.M.), University of Toronto, Toronto, Ontario, Canada

Received May 7, 2019; accepted August 1, 2019

\begin{abstract}
ORKAMBI, a combination of the corrector, lumacaftor, and the potentiator, ivacaftor, partially rescues the defective processing and anion channel activity conferred by the major cystic fibrosis-causing mutation, F508del, in in vitro studies. Clinically, the improvement in lung function after ORKAMBI treatment is modest and variable, prompting the search for complementary interventions. As our previous work identified a positive effect of arginine-dependent nitric oxide signaling on residual F508delCftr function in murine intestinal epithelium, we were prompted to determine whether strategies aimed at increasing arginine would enhance F508del-cystic fibrosis transmembrane conductance regulator (CFTR) channel activity in patient-derived airway epithelia. Now, we show that the addition of arginine together with inhibition of intracellular arginase activity increased cytosolic nitric oxide and enhanced the rescue effect of ORKAMBI on F508del-CFTR-mediated chloride conductance at the cell surface of patient-derived bronchial and nasal epithelial cultures.
\end{abstract}

Interestingly, arginine addition plus arginase inhibition also enhanced ORKAMBI-mediated increases in ciliary beat frequency and mucociliary movement, two in vitro CF phenotypes that are downstream of the channel defect. This work suggests that strategies to manipulate the arginine-nitric oxide pathway in combination with CFTR modulators may lead to improved clinical outcomes.

\section{SIGNIFICANCE STATEMENT}

These proof-of-concept studies highlight the potential to boost the response to cystic fibrosis (CF) transmembrane conductance regulator (CFTR) modulators, lumacaftor and ivacaftor, in patientderived airway tissues expressing the major CF-causing mutant, F508del-CFTR, by enhancing other regulatory pathways. In this case, we observed enhancement of pharmacologically rescued F508del-CFTR by arginine-dependent, nitric oxide signaling through inhibition of endogenous arginase activity.

\section{Introduction}

The approach to cystic fibrosis (CF) management has changed dramatically in recent years with the availability of CF transmembrane conductance regulator (CFTR) modulators, agents that directly target certain mutant versions

This work was supported by Cystic Fibrosis Canada and the Canadian Institute of Health Research [CF Canada Grant 3172; CIHR MOP-125855 and CIHR GPG-102171 to C.E.B.]. Patient-derived tissues were obtained through the Program for Individualized Cystic Fibrosis.

https://doi.org/10.1124/mol.119.117143.

S This article has supplemental material available at molpharm. aspetjournals.org. of the CFTR protein that cause CF. The potentiator, ivacaftor (VX-770), enhances the channel activity of certain rare mutant forms of CFTR that are properly processed to reach the cell surface but exhibit defective channel activity (Van Goor et al., 2009, 2014; Eckford et al., 2012; Yu et al., 2012; Jih and Hwang, 2013). Ivacaftor treatment alone is not effective for the major CF-causing mutation, deletion of phenylalanine at position 508 (F508del)-CFTR, because this mutant is misprocessed and mistrafficked and thus fails to reach the cell surface (Mall et al., 2004). The corrector compound, lumacaftor (VX-809), acts to partially correct this misprocessing and mistrafficking defect (Van Goor et al., 2011).

ABBREVIATIONS: ABH, 2(S)-amino-6-boronohexanoic acid; ACC, apical chloride conductance; ALI, air-liquid interface; CBF, ciliary beat frequency; CF, cystic fibrosis; CFTR, CF transmembrane conductance regulator; DAF-FM, 4-amino-5-methylamino-2' 7'-difluorofluorescein; FLIPR, fluorescent imaging plate reader; FSK, forskolin; HBSS, Hanks' buffered salt solution; iNOS, inducible NO synthase; MCCV, mucociliary movement; NO, nitric oxide; WT, wild-type. 
Lumacaftor combined with ivacaftor is called ORKAMBI, an approved intervention for patients who are homozygous for F508del (Wainwright et al., 2015). Unfortunately, ORKAMBI provides only modest and variable clinical responses in those patients (Cholon, et al., 2016; Zhang et al., 2016; Ratjen et al., 2017). Hence, there is an urgent need to develop novel therapeutic agents for the treatment of patients with F508del-CFTR, and there are a number of candidates proceeding through the drug development pipeline (https://www.cff.org/Trials/Pipeline).

Our previous studies in mice expressing F508del-Cftr (Cftr ${ }^{\text {F508del/F508del }}$ ) showed that elevations in cytosolic arginine increased nitric oxide (NO)-mediated signaling to the mutant Cftr protein to enhance its channel function (Ahmadi et al., 2018). We hypothesized that interventions aiming to enhance cytosolic arginine concentrations and NO signaling would also increase F508del-CFTR channel function in human respiratory epithelium. However, as human F508del-CFTR exhibits a more severe misprocessing defect relative to the mouse protein (Ostedgaard et al., 2007), the rescue effect of arginine would only be observed after correction of misprocessing and mistrafficking with lumacaftor.

Interestingly, our hypothesis converges with previous studies by Grasemann et al. (Grasemann et al., 2000; Grasemann and Ratjen, 2012; Grasemann and Pencharz, 2013). This group showed that CF patients exhibit a relative deficit in exhaled NO and attributed this deficiency to excessive arginase activity in pulmonary tissue (Grasemann et al., 2005b; Jaecklin et al., 2014). Citing early studies of the important role for argininedependent NO production (Grasemann et al., 1999) [via immune or inducible NO synthase (iNOS)] in the lung, including the host defense and the regulation of vascular and broncho-motor tone, Grasemann and colleagues conducted clinical trials of arginine or NO supplementation as therapeutic interventions for CF patients (Ratjen et al., 1999; Grasemann et al., 2013, 2005a). Unfortunately, neither NO inhalation nor arginine supplementation led to statistically significant improvements in forced expiratory volume at 1 second although promising positive trends were noted (Ratjen et al., 1999; Grasemann et al., 2005a, 2013).

In the current study, we tested the efficacy of arginase inhibitors, including CB-1158 [currently in clinical trial for cancer (ID: NCT02903914)], to augment NO signaling and ORKAMBI-mediated rescue of F508del-CFTR in vitro in primary cultures of patient-derived bronchial and nasal epithelial cultures.

\section{Materials and Methods}

Primary Bronchial Cell Culture. Primary bronchial cultures were obtained from non-CF and CF post-transplant bronchial tissue as part of a collaboration with S. Keshavjee (University of Toronto) and P. Karp and M. Welsh (National Institutes of Health Iowa Culture Facility). Cells were plated on collagen-coated transwells (6.5 mm diameter, $0.4 \mu \mathrm{m}$ pore size; Corning) and grown at an air-liquid interface (ALI). Media in the basolateral compartment was comprised of a 1:1 mixture of DMEM and Ham's F12 medium, supplemented with 2\% Ultroser G Serum Substitute (PALL France SAS). Transepithelial electrical resistance was measured with an ohmmeter (World Precision Instruments) and expected to be $>800 \Omega / \mathrm{cm}^{2}$ before any functional studies.

Primary Nasal Cell Culture. Nasal epithelial cells were obtained and cultured, as previously described, under local Research Ethics Board-approved protocols after subjects (or appropriate guardian) signed consent forms (Cao et al., 2015, 2018; Ahmadi et al., 2017). Frozen passage number 1 F508del-CFTR nasal cells were seeded on a collagen-coated flask and expanded at $37^{\circ} \mathrm{C}$ in a $5 \% \mathrm{CO}_{2}$ setting to 70\%-80\% confluence (PneumaCult EX; StemCell Tech). Passage number 3 cells were subsequently seeded in collagen-coated transwells ( $6.5 \mathrm{~mm}$ diameter, $0.4 \mu \mathrm{m}$ pore size; Corning) at a density of $10^{5}$ cells per insert. Cells were maintained in PneumaCult EX media until confluent and changed to a basal differentiation media (PneumaCult ALI; StemCell Tech) at an ALI. Media was changed every other day in the basolateral compartment. After 14 days of growth under ALI, transepithelial electrical resistance was quantified with an ohmmeter to evaluate barrier function. Cells were then used to measure CFTR function using the membrane potential assay.

For drug rescue experiments, nasal cells were treated with $3 \mu \mathrm{M}$ corrector VX-809 (Selleck Chemicals) for 48 hours.

Cell Line Culture. The CFBE cell line, overexpressing F508del CFTR (CFBE410-), was received from D. Gruenert (University of California) and maintained with Eagle's minimum essential medium with $10 \% \mathrm{FBS}, 1 \%$ penicillin-streptomycin, and $300 \mu \mathrm{g} / \mathrm{ml}$ hygromycin B. CFBE cells were plated in 96 -well black polystyrene plates and maintained for 4 to 5 days postconfluence for apical chloride conductance (ACC) assays. As CFBE cells do not express any apical arginine transporters, a broadly specific amino acid transporter, SLC6A14, was transduced into the cells by C. Tailor from Tailored Genes.

NO Measurement Assay. The NO measurement assay was performed on primary respiratory inserts with $700 \mu$ l Hanks' buffered salt solution (HBSS) containing chloride in the basolateral compartment of each well containing inserts in a 24 -well plate (Corning) and $200 \mu \mathrm{l} \mathrm{NaCl}$ buffer ( $145 \mathrm{mM} \mathrm{NaCl}, 3 \mathrm{mM} \mathrm{KCl}, 3 \mathrm{mM} \mathrm{CaCl}_{2}$, $2 \mathrm{mM} \mathrm{MgCl}_{2}, 10 \mathrm{mM}$ HEPES, $300 \mathrm{mOsm}, \mathrm{pH}$ 7.35) containing $7 \mu \mathrm{M}$ 4-amino-5-methylamino-2' 7'-difluorofluorescein (DAF-FM) diacetate (Cayman Chemical) and $10 \mu \mathrm{M}$ Calcein Blue AM (ThermoFisher Scientific) on the apical surface of the insert. The dye solution also contained chronic treatment in the form of $10 \mu \mathrm{M}$ CB1158 (MedKoo), $1 \mathrm{mM}$ L-arginine (Sigma-Aldrich), and/or the appropriate vehicle controls. After 1 hour of incubation of the dye solution, the cells were washed with PBS to remove excess dye and then incubated in dye-free $\mathrm{NaCl}$ buffer containing the aforementioned chronic treatments at $37^{\circ} \mathrm{C}$ for 20 minutes. Using the well scan endpoint feature of the i3x-Spectrophotometer (Molecular Devices), an initial live cell fluorescence scan at excitation $(322 \mathrm{~nm})$ and emission $(435 \mathrm{~nm})$ wavelengths was performed. Following that, NO fluorescence was measured at excitation $(495 \mathrm{~nm})$ and emission $(515 \mathrm{~nm})$ wavelengths. Sixty-four points in each well were read and averaged. After several baseline reads, $1 \mathrm{mM}$ L-arginine was added. As primary cell inserts are heterogeneous, final readout of DAF-FM fluorescence was calculated over Calcein Blue AM fluorescence. A minimum of three biologic replicates was used for primary insert studies.

ACC Assay for CFTR. Fluorescent imaging plate reader (FLIPR)based ACC assay was performed on the CFBE cell line and the primary respiratory cell ALI inserts (both bronchial and nasal) and were previously validated (Ahmadi et al., 2017).

CFBE cells were grown at $37^{\circ} \mathrm{C}$ to $100 \%$ confluence in 96-well (black, flat-bottom; Corning) plates and allowed to differentiate for 4 to 5 days. Following 48-hour incubation in the presence of the corrector $3 \mu \mathrm{M}$ VX-809 (Selleck Chemicals) at $27^{\circ} \mathrm{C}$, the cells were placed in FLIPR blue membrane potential dye (Molecular Devices) at a concentration of $0.5 \mathrm{mg} / \mathrm{ml}$ dissolved in buffer (112.5 mM N-methyl-Dglucamine-gluconate, $36.25 \mathrm{mM} \mathrm{NaCl}, 2.25 \mathrm{mM}$ K.gluconate, $0.75 \mathrm{mM}$ $\mathrm{KCl}, 0.75 \mathrm{mM} \mathrm{CaCl}_{2}, 0.5 \mathrm{mM} \mathrm{MgCl}_{2}$, and $10 \mathrm{mM} \mathrm{HEPES}$, and $300 \mathrm{mOsm}$, $\mathrm{pH}$ 7.35) supplemented with $3 \mu \mathrm{M}$ VX-809, to detect changes in transmembrane potential of the differentiated cells. Pretreatment with $10 \mu \mathrm{M}$ CB-1158 (MedKoo) and/or $50 \mu \mathrm{M} 1400 \mathrm{~W}$ (Cayman Chemical) was tested. As previously described (Molinski et al., 2015), following a 40-minute incubation period of the dye solution, a kinetic read of the plate was made in a fluorescence plate reader (SpectraMax i3; Molecular Devices) at $27^{\circ} \mathrm{C}$. After reading the 
baseline fluorescence (530/560 $\mathrm{nm}$ excitation emission wavelengths), $1 \mathrm{mM}$ L-arginine was added to the cells with buffer addition as a parallel control. CFTR was stimulated using $10 \mu \mathrm{M}$ forskolin (FSK; Sigma-Aldrich) with DMSO vehicle as the negative control. The experiment was terminated with CFTR-specific inhibitor $10 \mu \mathrm{M}$ CFTRinh-172 (EMD Millipore). Changes in transmembrane potential were normalized to the point taken at the time of agonist addition prior to activation. Four biologic replicates were used for the ACC assay studying these CFBE cell lines.

For primary ALI inserts, the FLIPR-based ACC assay was performed differently than CFBE cells. Following 48-hour incubation in the presence of $3 \mu \mathrm{M}$ corrector VX- 809 at $37^{\circ} \mathrm{C}$, the basal and apical solutions were kept different in the 24-well plates (Corning); the apical solution of the insert contained $0.5 \mathrm{mg} / \mathrm{ml}$ blue membrane potential dye (Molecular Devices) in buffer (112.5 mM N-methyl-D-glucamine-gluconate, $36.25 \mathrm{mM} \mathrm{NaCl}, 2.25 \mathrm{mM}$ K-gluconate, $0.75 \mathrm{mM} \mathrm{KCl}, 0.75 \mathrm{mM}$ $\mathrm{CaCl}_{2}, 0.5 \mathrm{mM} \mathrm{MgCl}_{2}$, and $10 \mathrm{mM}$ HEPES, and $300 \mathrm{mOsm}, \mathrm{pH} 7.35$ ), whereas the basal side contained Hanks' buffered solution containing chloride. In the apical dye solution, pretreatment with $10 \mu \mathrm{M} \mathrm{ABH}$ (2(S)-amino-6-boronohexanoic acid) ammonium salt (Santa Cruz), $10 \mu \mathrm{M}$ CB-1158, or DMSO controls was added prior to incubation. After 30 - minute incubation at $37^{\circ} \mathrm{C}$, the fluorescent readings at excitation wavelength $(530 \mathrm{~nm})$ and emission wavelength $(560 \mathrm{~nm})$ were quantified with a micro-plate reader (Paradigm; Molecular Devices). Sixty-four points in each well were read, averaged, and thresholded. After several baseline reads, pharmacological modulators ( $1 \mathrm{mM}$ L-arginine or the respective vehicle controls) were added, followed by $10 \mu \mathrm{M}$ FSK (Sigma-Aldrich) $\pm 1 \mu \mathrm{M}$ VX-770 or DMSO

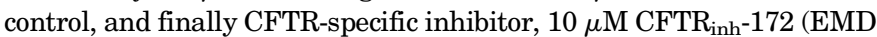
Millipore). A minimum of three separate patient tissue samples was used for each demonstrated condition.

Upon completion of the ACC experiments, the raw data were exported and analyzed using the hiflo.xyz analysis platform.

Ussing Chamber Studies. F508del-CFTR Nasal cells were pretreated with $3 \mu \mathrm{M}$ VX-809 for 48 hours. Prior to the start of the Ussing chamber recordings, nasal cells were pretreated with $10 \mu \mathrm{M}$ CB-1158 or DMSO for 1 hour. Drugs for chronic or pretreatment were added to the culture media on the basolateral side of the transwell insert. Nasal cells were studied in a nonperfused Ussing chamber (Physiologic Instruments). The buffer (126 mM NaCl, $24 \mathrm{mM} \mathrm{NaHCO}, 2.13 \mathrm{mM}$ $\mathrm{K}_{2} \mathrm{HPO}_{4}, 0.38 \mathrm{mM} \mathrm{KH}_{2} \mathrm{PO}_{4}, 1 \mathrm{mM} \mathrm{MgSO}{ }_{4}, 1 \mathrm{mM} \mathrm{CaCl}_{2}$, and $10 \mathrm{mM}$ glucose) was maintained at $\mathrm{pH} 7.4$ and $37^{\circ} \mathrm{C}$ and continuously gassed with $5 \% \mathrm{CO}_{2} / 95 \% \mathrm{O}_{2}$ mix (Galietta et al., 1998). The transepithelial potential was recorded and the baseline resistance was measured following repeated, brief short-circuit current pulses (1 $\mu \mathrm{A}$ every 30 seconds). A chloride gradient was established for the nasal cells by replacing the buffer on the apical side with a low chloride buffer (1.2 mM NaCl, $115 \mathrm{mM} \mathrm{Na}$ gluconate, $2.4 \mathrm{mM} \mathrm{KH} \mathrm{KO}_{4}, 1.24 \mathrm{mM}$ $\mathrm{KH}_{2} \mathrm{PO}_{4}, 1 \mathrm{mM} \mathrm{MgCl} 2,1 \mathrm{mM} \mathrm{CaCl}, 25 \mathrm{mM} \mathrm{NaHCO}_{3}$, and $10 \mathrm{mM}$ glucose).

The results are presented as the calculated short-circuit transepithelial current $\left(\mu \mathrm{A} / \mathrm{cm}^{2}\right)$. CFTR function was determined after inhibition of the epithelial sodium channel with amiloride $(30 \mu \mathrm{M}$; Spectrum Chemical) and cAMP activation with $10 \mu$ M FSK (SigmaAldrich). CFTR activity was calculated as short-circuit transepithelial current difference following CFTR inhibition with $10 \mu \mathrm{M}$ CFTRinh-172 (EMD Millipore). Depending on the conditions, $1 \mathrm{mM}$ L-arginine was added to observe the combinational effect of arginine with specific drugs added during pretreatment. Three biologic replicates were used for this Ussing chamber study.

Immunoblotting. Nasal cultures were grown at $37^{\circ} \mathrm{C}$ for 48 hours in the presence of $3 \mu \mathrm{M}$ VX-809. One hour before the experiments, the cells were treated with $10 \mu \mathrm{M}$ CB-1158 drug or DMSO control, as required. Cells were then lysed in modified radioimmunoprecipitation assay buffer [50 mM Tris-HCl, $150 \mathrm{mM} \mathrm{NaCl}, 1 \mathrm{mM}$ EDTA, $\mathrm{pH}$ 7.4, $0.2 \%(\mathrm{v} / \mathrm{v})$ SDS, and $0.1 \%(\mathrm{v} / \mathrm{v})$ Triton X-100] containing a protease inhibitor cocktail (Roche) for 10 minutes, and the soluble fractions were analyzed by SDS-PAGE on $6 \%$ gels, as described previously

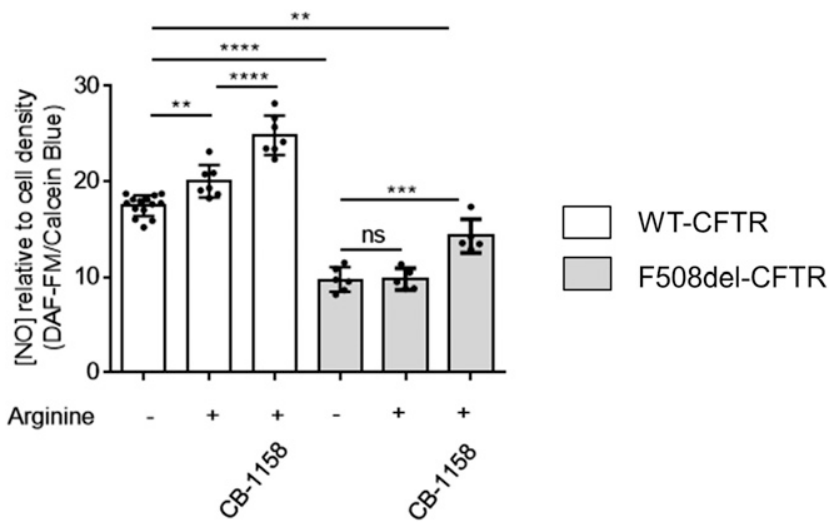

Fig. 1. CF-associated defect in arginine-dependent NO production in bronchial epithelia is partially rescued by the arginase inhibitor, CB-1158. Epithelial NO levels were measured using DAF-FM fluorophore in WTand F508del-CFTR primary bronchial cells following simultaneous 1-hour pretreatment with $1 \mathrm{mM}$ L-arginine $\pm 10 \mu \mathrm{M} \mathrm{CB}-1158$ and the appropriate buffer control. Calculations were generated by normalizing the NO concentration to cell density (the ratio of DAF-FM to the live cell marker, Calcein Blue AM) to compare between the two patient genotypes. Bars represent mean \pm S.D. One-way ANOVA with Tukey's multiple comparison test was performed. $(\mathrm{ns}=$ not statistically significant; $* * P<0.01$; $* * * P<0.002 ; * * * * P<0.0001, N>4$ biologic replicates, $n=2$ to 3 technical replicates).

(Laselva et al., 2018a; Molinski et al., 2018). After electrophoresis, proteins were transferred to nitrocellulose membranes and incubated in $5 \%(\mathrm{w} / \mathrm{v})$ milk, and CFTR bands were detected using the human CFTR-NBD2-specific muring mAb 596 (1:500; University of North Carolina Chapel Hill). Connexin was used as a loading control and detected using a connexin-specific rabbit Ab (1:5000; SigmaAldrich). Blots were exposed with Amersham Biosciences enhanced chemiluminescent reagent (GE Healthcare) on the Li-Cor Odyssey Fc (LI-COR Biosciences, Lincoln, NE) in a linear range of exposure (2 to 20 minutes) (Chin et al., 2017; Laselva et al., 2018b).

Fluorescent Bead-Tracking Assay. Twenty-four hours prior to bead-tracking assay, primary inserts were gently washed on the apical surface and incubated in HBSS for 1 hour, before cleared of any apical fluids. One hour prior to the experiment, $50 \mu \mathrm{l}$ fluorescent probe suspension in HBSS, consisting of FluoSpheres Polystyrene Microspheres $1.0 \mu \mathrm{m}$, green fluorescent or red fluorescent (ThermoFisher Scientific) at $0.02 \%$ was placed onto the apical surface of the inserts. In the fluorescent probe suspension, pretreatment was added as well, which included $10 \mu \mathrm{M}$ CB-1158, $10 \mu \mathrm{M} \mathrm{ABH}$, or the respective vehicle controls.

Methods were adapted from Matsui et al. (1998). All experiments were conducted at $37^{\circ} \mathrm{C}$ with $5 \% \mathrm{CO}_{2}$. Mucus transport rates over the surface of the planar cultures were measured from videos (5 seconds) at $10 \times$ and $20 \times$ magnifications with an inverted epifluorescence microscope with a temperature control cage (Nikon eclipse TE2000) and a Hamamatsu orca ER camera capable of taking images at a rate of 9 to 12 frames per second. A baseline recording of bead movement was conducted for each cell monolayer prior to apical administration of a $2 \mu \mathrm{l}$ test solution containing DMSO control, $10 \mu \mathrm{M}$ FSK, or $10 \mu \mathrm{M}$ FSK with $1 \mu \mathrm{M}$ VX-770. Thirty minutes after addition of test solution, $10 \times$ and $20 \times$ magnification videos were taken again, focusing on the fluorescent beads. Five videos were taken per magnification in the center of the insert as well as in the north, south, east, and west quadrants. Upon completion of each set of experiments, the fluorescent beads of each video were enhanced via the contrast enhancement tool and exported as TIFF files in Volocity 6.3. The tracking software, Arivis vision4d, was used to analyze each individual bead velocity and speed using a predetermined tracking pipeline, used to analyze 3 seconds of imported TIFF files ( $\geq 27$ images) with the top 25 percentile of fluorescence intensity, and exported as an excel file. 
A

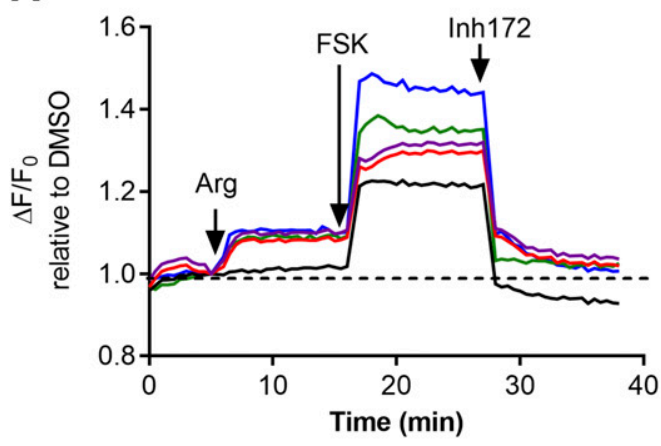

B

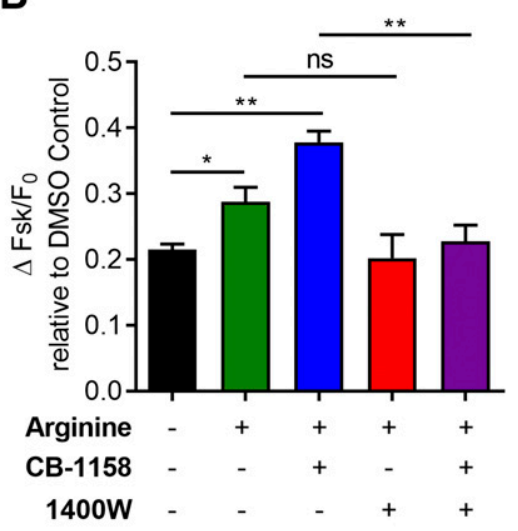

Fig. 2. Pretreatment with arginase inhibitor CB-1158 increases NO pathway-driven CFTR channel activity in CFBE cells transduced with lv (lentivirus). SLC6A14. (A) Traces normalized to 5 minutes (timepoint at the end of baseline) represent change in FLIPR fluorescence from baseline (depolarization due to ACC: $\triangle \mathrm{F} / \mathrm{F}_{0}$ ) relative to buffer control, as a measure of CFTR channel function in CFBE stably expressing F508del-CFTR with lv. SLC6A14. Cells were pretreated 1 hour with $10 \mu \mathrm{M}$ CB-1158, $50 \mu \mathrm{M} 1400 \mathrm{~W}$, combination of CB-1158 and $1400 \mathrm{~W}$, or buffer in the FLIPR dye solution. At the 5-minute point, $1 \mathrm{mM}$ L-arginine was added into the apical bath, and, at 15 minutes, cAMP stimulation occurred due to $10 \mu \mathrm{M}$ FSK addition. At 25 minutes, CFTR-specific inhibitor, CFTR ${ }_{\text {inh }}-172$ (Inh-172), was added to confirm CFTR-specific function. (B) Bar graph represents maximum change in ACC fluorescence after Fsk addition to the peak of Fsk response $\left(\Delta \mathrm{Fsk} / \mathrm{F}_{0}\right)$ following $1 \mathrm{mM}$ L-arginine in the CFBE cell line from (A) (mean $\pm \mathrm{S} . D$.). Oneway ANOVA with Tukey's multiple comparison test was performed (ns $=$ not statistically significant, $* P<0.05 ; * * P<0.01, N=3$ biologic replicates, $n=4$ technical replicates).

The following formula was used to generate the outputted tracked displacement (meters) and to determine the velocity of the beads.

\section{$\mid$ Velocity $\mid(\mu \mathbf{m} / \mathbf{s})=\operatorname{displacement}(\mathbf{m}) \times 10^{6}$ \\ $\times(\text { the number of frames the beads were observed in })^{-1}$ \\ $\times$ (camera speed in frames per second $)$}

The displacement values were multiplied by $10^{6}$ to convert meters to micrometers. The excel file-generated "the number of frames that the beads were observed in" was used to generate the individual frame-to-frame velocity and then multiplied by the camera speed in frames per second to generate the velocity per unit of time in seconds. The reported values describe the arithmetic means of these values. Analysis was either given as the velocity in micormeters per second or normalized to fold-change over baseline.

Mucociliary Beat Frequency Assay. Twenty-four hours prior to bead-tracking assay, primary inserts were gently washed apically and incubated in HBSS for 1 hour, and then cleared of all apical fluids. One hour prior to experiment, $50 \mu \mathrm{l}$ HBSS suspension, containing pretreatment, was added, which included $10 \mu \mathrm{M} \mathrm{CB}-1158,10 \mu \mathrm{M} \mathrm{ABH}$, or the respective vehicle controls.

All experiments were conducted at room temperature for imaging and incubated at $37^{\circ} \mathrm{C}$ with $5 \% \mathrm{CO}_{2}$ pre- and post-treatment. Ciliary beat frequency $(\mathrm{CBF})$ assay was conducted at $40 \times$ magnification using oil immersion on the inverted epifluorescence microscope Zeiss Axiovert $200 \mathrm{~m}$ with a high-speed monochromatic digital camera (Hamamatsu orca flash 4.0) at a sampling rate of $>120$ frames per second and a resolution of $1280 \times 720$ pixels of the ciliary layer from an en face viewpoint. A baseline recording of $\mathrm{CBF}$ was conducted for each cell monolayer prior to apical administration of a $2 \mu \mathrm{l}$ test solution containing DMSO control, $10 \mu \mathrm{M}$ FSK, or $10 \mu \mathrm{M}$ FSK with $1 \mu \mathrm{M}$ VX770. Thirty minutes after addition of the test solution, $40 \times$ magnification videos were taken again, focusing on the ciliary layer. Five videos were taken per magnification in the center of the insert as well as in the north, south, east, and west quadrants. Upon completion of each set of experiments, the ciliary layer of each video was enhanced via the contrast enhancement tool and exported as TIFF files in
Volocity 6.3. Video images were analyzed using MatLab application program Cilia Beat Analyzer developed by J. Chen (University of California-Irvine) (Chen et al., 2016). The reported frequencies were derived as the peak response of the Gaussian distribution as derived using GraphPad Prism 7.0. Analysis was given as the raw Hertz values generated.

Statistical Analysis. One-way ANOVA with Tukey's multiple comparison test was performed on all data with more than two datasets for comparison. S.D. was calculated using data from biologic replicates from patients and the average of technical replicates per plate from cell lines. Unpaired two-tailed $t$ test was performed on data with two data sets. $P<0.05$ was considered statistically significant. Statistical analyses were performed using GraphPad Prism 8.

\section{Results}

Addition of Apical Arginine Plus Inhibitors of Arginase-Enhanced NO Production in Primary Bronchial Cultures Obtained from Non-CF and CF Donors. In our previous work, we showed that arginine is transported across the apical membrane of murine intestinal epithelia and converted to NO (Ahmadi et al., 2018). Now, we show that arginine uptake across the apical membrane of primary bronchial cultures (from nine donors) also leads to an increase in cytosolic NO in non-CF cultures (Fig. 1, white bars). As expected, the fluorescence signal, conferred by DAF-FM and corresponding to cytosolic NO, increased after the apical addition of L-arginine $(1 \mathrm{mM})$ alone, and when arginine addition was combined with addition of either competitive arginase inhibitors, ABH $(1 \mu \mathrm{M})$ or CB-1158 $(10 \mu \mathrm{M})$, compounds that are expected to inhibit both forms of arginase (I and II) (Steggerda et al., 2017; van den Berg et al., 2018). The structures of these compounds are shown in Supplemental Fig. 1. 

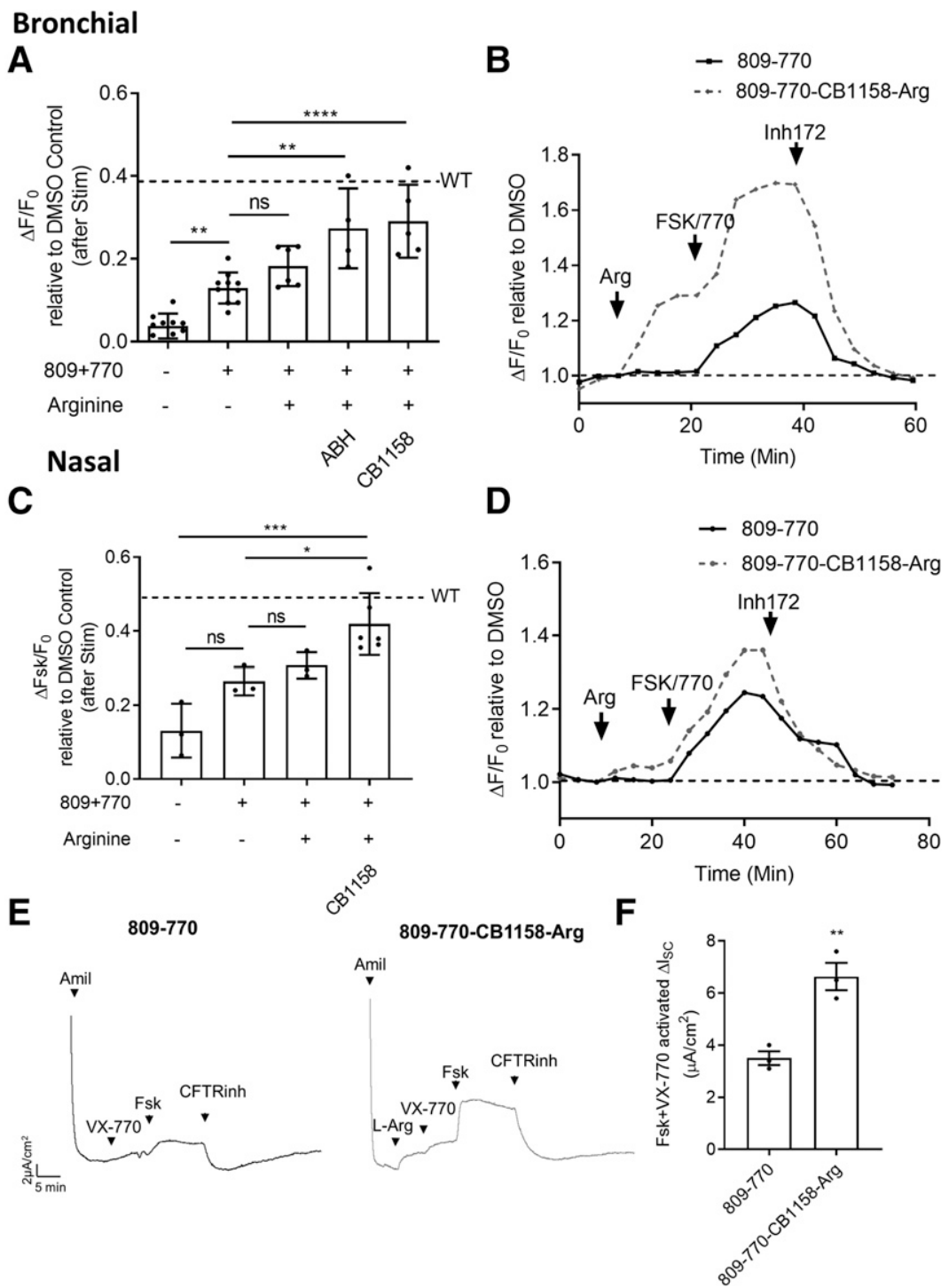

Fig. 3. Inhibition of arginase activity enhances rescue of F508del-CFTR channel activity at the surface of primary respiratory epithelial tissues by VX809 plus VX-770. (A) Bar graph represents maximum change in ACC fluorescence from baseline $\left(\Delta \mathrm{F} / \mathrm{F}_{0}\right)$ normalized to mock activation (with DMSO as vehicle control for arginine or FSK plus VX-770) for F508del-CFTR primary bronchial cells with 48-hour chronic treatment with either DMSO or $3 \mu \mathrm{M}$ VX-809 (mean \pm S.D.). Cells were pretreated acutely for 1 hour with $1 \mathrm{mM}$ L-arginine, $10 \mu \mathrm{M} \mathrm{CB}-1158,10 \mu \mathrm{M}$ ABH, or buffer in the FLIPR dye solution. WT baseline is based on the mean FSK-stimulated values of eight individual WT-CFTR primary bronchial cells. One-way ANOVA with Tukey's multiple comparison test relative to VX-809 plus VX-770 was performed (**P<0.007; **** $P<0.0001, N>4$ biologic replicates, $n=2$ technical replicates). (B) Representative line graphs represent change in ACC fluorescence from baseline $\left(\Delta \mathrm{F} / \mathrm{F}_{0}\right)$ relative to DMSO control, as a measure of CFTR channel function in F508del-CFTR bronchial cells grown on inserts at ALI with 48-hour chronic treatment of $3 \mu \mathrm{M}$ VX-809 or DMSO. Cells were pretreated with $10 \mu \mathrm{M}$ CB1158 , or buffer in the FLIPR dye solution. At the 10-minute point, $1 \mathrm{mM}$ L-arginine (Arg) was added into the apical FLIPR membrane dye solution, and, at 25 minutes, cAMP stimulation (Stim) occurred due to $10 \mu \mathrm{M}$ FSK and $1 \mu \mathrm{M}$ VX-770 addition. At 45 minutes, CFTR-specific inhibitor, CFTR inh-172 (Inh172), was added as an indicator of CFTR-specific function. (C) Bar graph represents the maximum change in ACC fluorescence from baseline $\left(\Delta \mathrm{F} / \mathrm{F}_{0}\right)$ normalized to first DMSO control on F508del-CFTR primary nasal cells with 48-hour chronic treatment of either DMSO or $3 \mu$ M VX-809 (mean \pm S.D.). Cells are pretreated for 1 hour with $10 \mu \mathrm{M} \mathrm{CB}-1158$, or buffer in the FLIPR dye solution. WT baseline is based on the mean FSK-stimulated values of 16 individual WT-CFTR primary nasal cells. One-way ANOVA with Tukey's multiple comparison test was performed (ns = not statistically significant, ${ }^{*} P<0.04 ; * * P=0.005, N=3$ biologic replicates, $n=1$ to 2 technical replicates). (D) Representative trace shows change in ACC fluorescence from baseline $\left(\Delta \mathrm{F} / \mathrm{F}_{0}\right)$ relative to DMSO control, as a measure of CFTR channel function in F508del-CFTR nasal cells grown on inserts at ALI for 14 days with 48 -hour chronic treatment of $3 \mu \mathrm{M}$ VX-809 or DMSO. Cells were pretreated with $10 \mu \mathrm{M}$ CB-1158 or buffer in the FLIPR dye solution. At the 10 -minute point, $1 \mathrm{mM}$ L-arginine (Arg) was added into the apical buffer, and, at 25 minutes, cAMP stimulation (Stim) occurred due to $10 \mu \mathrm{M}$ FSK and $1 \mu \mathrm{M}$ VX-770

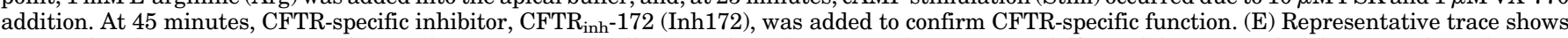
Ussing chamber measurements of CFTR function in nasal cell culture from CF patients bearing F508del/F508del pretreated with VX-809 for 48 hours in absence (left) or presence of CB-1158 plus arginine $(1 \mathrm{mM})$ for 1 hour (right). CFTR function was determined after inhibition of the epithelial sodium channel (ENaC) with $30 \mu \mathrm{M}$ amiloride and cAMP activation with $10 \mu \mathrm{M}$ FSK. The subsequent addition included $10 \mu \mathrm{M}$ CFTRinh-172 to determine CFTR-specific activity. (F) Bar graph showing mean ( \pm S.D.) FSK and VX-770-activated short-circuit transepithelial current $\left(\mathrm{I}_{\mathrm{SC}}\right)$ for nasal cultures from three patients bearing F508del/F508del mutation, after pretreatment of 48 hours with VX-809 $(3 \mu \mathrm{M}) \pm$ CB-1158 $(1 \mu \mathrm{M})(\mathrm{mean} \pm \mathrm{S}$.D.). Unpaired $t$ test was performed ( $* P=0.0059, N=3$ biologic replicates, $n=1$ technical replicate). For VX-809 or VX-809 plus CB1158 pretreatment, the confidence limits were $2.4-4.4$ and $4.6-8.9 \mu \mathrm{A} / \mathrm{cm}^{2}$, respectively, using a confidential level of $95 \%$. 
Interestingly, in bronchial cultures generated from a total of nine donors who are homozygous for F508del, the basal levels of NO were significantly less, statistically, than those measured in the non-CF cultures, and there was no increase in these basal levels after the addition of apical arginine unless the arginase inhibitor CB-1158 was also added (Fig. 1). These findings are consistent with previous reports that $\mathrm{CF}$ bronchial epithelial cells exhibit elevated arginase activity relative to non-CF (Grasemann et al., 2005b; Jaecklin et al., 2014). Although the transport of arginine in the presence of arginase inhibitors led to an increase in cytosolic NO in the F508del cultures, NO levels were not fully rescued to those measured in basal conditions in non-CF cultures (Fig. 1).

We showed previously in our studies of intestinal epithelium that arginine-mediated NO generation enhanced the channel activity of wild-type (WT)-CFTR and F508del-CFTR, after rescue of its trafficking defect using lumacaftor or VX809 (Ahmadi et al., 2018). In this study, we confirmed that a similar augmentation occurs in $\mathrm{CF}$ bronchial epithelium provided by D. Gruenert (University of California), namely the cell line: CFBE410 ${ }^{-}$stably overexpressing F508delCFTR (Treharne et al., 2008). In Fig. 2, we show, using the plate-based, fluorescence assay of F508del-CFTR-mediated chloride conductance (Molinski et al., 2015; Ahmadi et al., 2017), that in VX-809-corrected cells, after electrogenic uptake of arginine, there is an increase in the FSK-mediated response by the mutant CFTR. This increase is enhanced by the pretreatment with the arginase inhibitor: CB-1158. Importantly, and as expected for a NO-mediated effect, both the arginine and CB1158 effects on the FSK response were inhibited by pretreatment with the iNOS inhibitor, $1400 \mathrm{~W}$ (Ahmadi et al., 2018). Together, these results suggest that the regulated function of corrected F508del-CFTR in airway cells is augmented by NO generated after arginine accumulation intracellularly and iNOS activation, as shown in the model depicted in Fig. 8.

VX-809 Plus VX-770-Mediated Rescue of F508del-CFTR Channel Activity in Primary Bronchial and Nasal Epithelial Cultures Was Enhanced by Apical Arginine Plus Inhibitors of Arginase. Because we found previously that arginine-dependent $\mathrm{NO}$ signaling enhanced the function of residual F508del-Cftr protein in the intestinal epithelium of mice (Ahmadi et al., 2018), we were prompted to determine whether arginine-dependent NO signaling also enhanced F508del-CFTR protein in human bronchial and nasal epithelial cultures.

In Fig. 3, A and B, we show the results of studies on cultures generated from a minimum of four different patients (homozygous for F508del), each with two technical replicates.

First, we compared the effects of multiple interventions simultaneously in a multiwell assay of primary bronchial tissues using the fluorescence-based FLIPR method for measuring CFTR-mediated changes in membrane potential (ACC or the ACC assay) (Ahmadi et al., 2017). As shown in the bar graph (Fig. 3A), as expected, a statistically significant rescue of F508del-CFTR-mediated channel function, or ACC: $\Delta \mathrm{F} / \mathrm{F}_{0}$, was measured after 48 -hour pretreatment with the corrector $(3 \mu \mathrm{M}$ VX-809) and stimulation with FSK $(10 \mu \mathrm{M})$ and the potentiator $(1 \mu \mathrm{M}$ VX-770). The acute addition of L-arginine $(1 \mathrm{mM})$ to the apical surface of VX-809-corrected cultures led to a trend toward greater depolarization of the apical membrane following stimulation, as expected for this electrogenic transporter

\section{Bronchial}

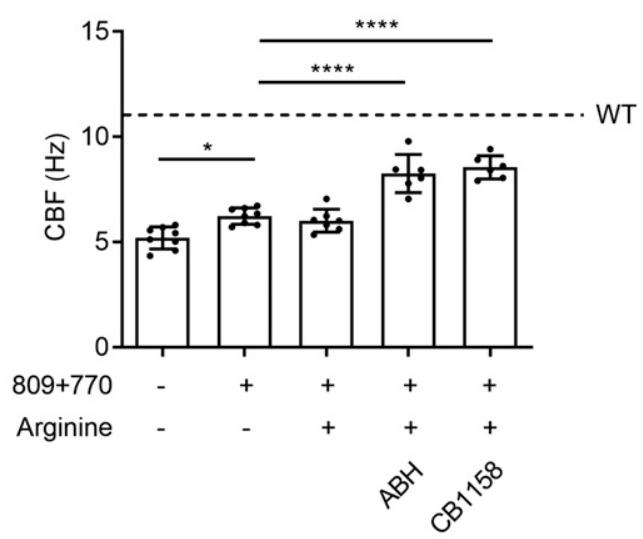

Nasal

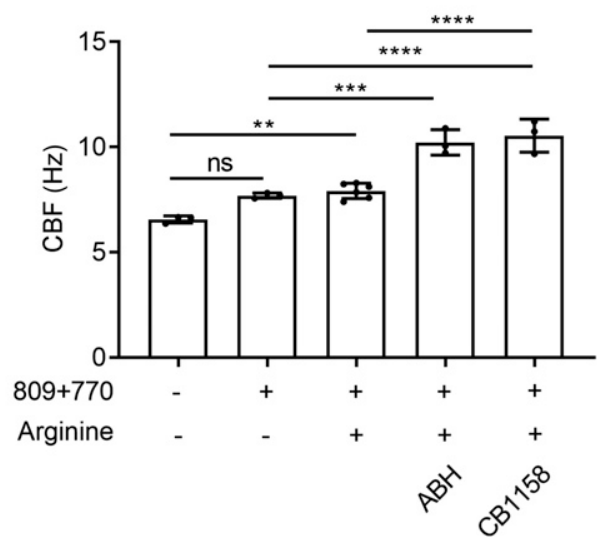

Fig. 4. Inhibition of arginase activity enhances rescue of $\mathrm{CBF}$ at the surface of primary respiratory epithelial tissues by VX-809 plus VX-770. Top panel: Bar graph represents CBF (Hertz) on F508del-CFTR primary bronchial cells with 48-hour chronic treatment of either DMSO or $3 \mu \mathrm{M}$ VX-809 (mean \pm S.D.). Cells were pretreated with $1 \mathrm{mM}$ L-arginine, $10 \mu \mathrm{M}$ CB-1158, $10 \mu \mathrm{M}$ ABH, or buffer in the HBSS solution following 1 hour of equilibration and 30 minutes of either $10 \mu \mathrm{M}$ FSK (first bar) or $10 \mu \mathrm{M}$ FSK with $1 \mu \mathrm{M}$ VX-770 (remaining bars) apical addition to the HBSS. WT baseline is based on the mean FSK-stimulated values of eight individual WT-CFTR primary bronchial cells. One-way ANOVA with Tukey's multiple comparison test was performed when comparing the first bar (VX$809+$ VX-770) with the rest of the conditions $(* P<0.05 ; * * * P<0.0001$, $N>3$ biologic replicates, $n=2$ technical replicates). Bottom panel: Bar graph represents FSK-stimulated CBF (Hertz) of the fully differentiated (day 30 postseeding) F508del-CFTR primary nasal cells with 48-hour chronic treatment of either DMSO or $3 \mu \mathrm{M}$ VX-809 (mean \pm S.D.). Cells were pretreated with $1 \mathrm{mM}$ L-arginine, $10 \mu \mathrm{M}$ CB-1158, $10 \mu \mathrm{M} \mathrm{ABH}$, or buffer in the HBSS solution following 1 hour of equilibration and 30 minutes of either $10 \mu \mathrm{M}$ FSK (first bar) or $10 \mu \mathrm{M}$ FSK with $1 \mu \mathrm{M}$ VX770 (remaining bars) apical addition to the HBSS. One-way ANOVA with Tukey's multiple comparison test was performed $(\mathrm{ns}=$ not statistically significant, ${ }^{* * *} P=0.001 ; * * * * P<0.0001, N=3$ biologic replicates, $n=2$ technical replicates).

(Ahmadi et al., 2018). But, as clear from the bar graph of Fig. 3A, arginine addition alone did not enhance the stimulation caused by FSK plus VX-770. In contrast, if arginine was added together with inhibitors of arginase, either ABH or CB1158, there was almost a 1.5 -fold enhancement of the stimulated channel function. Together with the results shown in Figs. 1 and 2, these results suggest that a positive effect of argininedependent NO signaling to surface-localized F508del-CFTR channels requires simultaneous inhibition of arginine metabolism. In Fig. 3B, we show a representative ACC trace, 
A

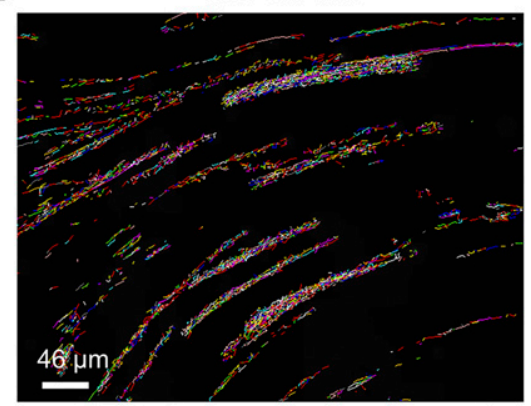

F508del-CFTR

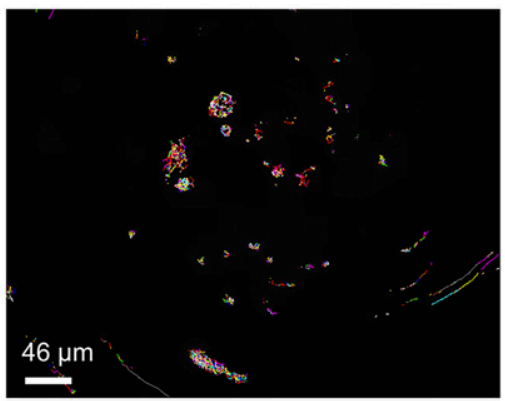

B

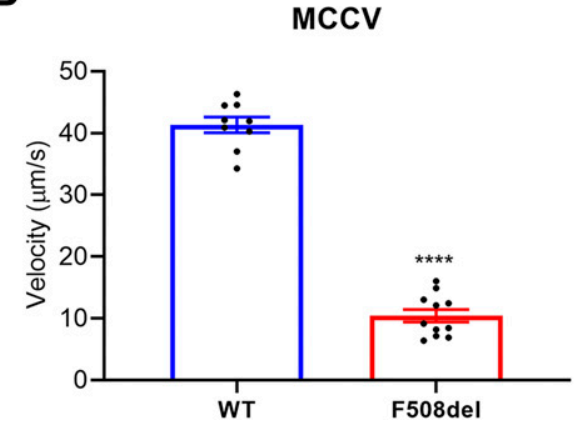

Fig. 5. Velocity and pattern of bead movement are defective in F508del respiratory cultures. (A) Left: Representative MCCV of a WT bronchial epithelial insert. Each color represents an individual bead movement over the course of 10 seconds following 1-hour equilibration of WTCFTR bronchial epithelial inserts in HBSS solution containing $0.02 \%$ polystyrene microspheres. MCCV appears as a mostly homogenous circular motion. Right: Representative MCCV of a F508del-CFTR bronchial epithelial insert shows less movement of each individual bead compared with the WT bronchial epithelial insert. (B) Bar graph represents the raw MCCV (micrometers per second) of either WT-CFTR or F508del-CFTR primary bronchial cells with 48-hour chronic treatment of DMSO (mean \pm S.D.). Cells were pretreated with buffer in the HBSS solution containing $1 \mu \mathrm{m}$ green or red polystyrene microspheres $(0.02 \%)$ following 1 hour of equilibration. Unpaired $t$ test was performed $(* * * * P<0.0001, N>8$ biologic replicates, $n=1$ technical replicate). corresponding to F508del-CFTR channel activity stimulated by FSK and VX-770 after correction (3 $\mu \mathrm{M}$ VX-809) in the presence or absence of arginine plus the arginase inhibitor CB-1158 (10 $\mu \mathrm{M})$.

Nasal epithelial cultures (differentiated at ALI) are an accepted in vitro model of patient-dependent responses to CFTR modulators (de Courcey et al., 2012; Müller et al., 2013; Cao et al., 2015), and thus, we conducted a similar set of studies in patient-derived nasal epithelial cultures. In this case, we compared ACC responses in cultures generated from three different patients with one to two technical replicates. In this study, acute arginine addition led to a modest depolarization compared with that observed in the bronchial cultures. But, similar to the responses observed in the bronchial cultures, the combination of apical arginine plus an arginase inhibitor enhanced the functional rescue mediated by FSK plus VX-770 in VX-809-corrected cultures by approximately 1.5-fold (Fig. 3, C and D). Addition of the arginase inhibitor, in the absence of added arginine, was not sufficient to enhance the response to VX-809 plus VX-770 (Supplemental Fig. 2), supporting the idea that apical uptake of arginine provides necessary substrate on which the arginase acts. Together with the results shown in Fig. 1, these studies suggest that NO generated by L-arginine in the presence of arginase inhibition further enhances potentiated channel activity of F508delCFTR after its biosynthetic correction.

Having determined that arginine addition together with an arginase inhibitor like CB1158 was effective in augmenting stimulated F508del-CFTR channel function after biosynthetic rescue with VX-809 in nasal epithelial cultures using the FLIPR assay, we were prompted to confirm this effect using the lower throughput Ussing chamber assay. Representative short-circuit traces for nasal cultures generated from the same CF individual homozygous for F508del-CFTR are shown. These cultures were corrected using VX-809 and then stimulated with FSK plus VX-770 alone or together with arginine plus arginase inhibitor, as shown in Fig. 3E. It is clear, from the comparison, that the addition of arginine plus CB1158 augments the functional stimulation of mutant CFTR channel activity in corrected cultures. We confirmed that this increase was not due to a change in F508del-CFTR expression or processing by immunoblotting (Supplemental Fig. 4). This enhancement was observed for a total of three paired cultures, each derived from a different individual (Fig. 3F). Interestingly, there was no statistically significant effect of arginine plus CB1158 on amiloride-sensitive currents in these experiments.

VX-809 Plus VX-770-Mediated Rescue of CBF and Mucociliary Movement in Primary CF Epithelial Cultures Were Enhanced by Apical Arginine Plus Inhibitors of Arginase. We then asked whether apical arginine and arginase inhibition improved $\mathrm{CBF}$ and mucociliary movement (MCCV) in CF airway cultures, as both of these in vitro phenotypes are thought to reflect CFTR channel function. First, we measured $\mathrm{CBF}$ of non-CF bronchial cultures in the presence of FSK $(10 \mu \mathrm{M})$. In the presence of vehicle alone, CBF is approximately $8 \mathrm{~Hz}$, which can be increased to $10 \mathrm{~Hz}$ with FSK (Supplemental Fig. 3). This change in CBF is consistent with values that were reported previously (Devalia et al., 1990; Sutto et al., 2004; Chen et al., 2016).

Then we assessed changes in CBF in differentiated bronchial and nasal epithelial cultures derived from patients homozygous for the F508del mutation. In the case of untreated (no VX-809 or VX-770) bronchial cultures ( $n \geq 6$ patients per treatment condition), FSK-dependent CBF was approximately $50 \%$ of the $\mathrm{CBF}$ observed in the non-CF cultures. Following VX-809 plus VX-770 rescue, CBF increased significantly (Fig. 4, top). Pretreatment with arginine $(1 \mathrm{mM})$ for 1 hour did not significantly increase CBF relative to that observed in VX809-corrected and VX-770-potentiated cultures. Interestingly, the combination of arginine plus arginase inhibition 


\section{Bronchial}

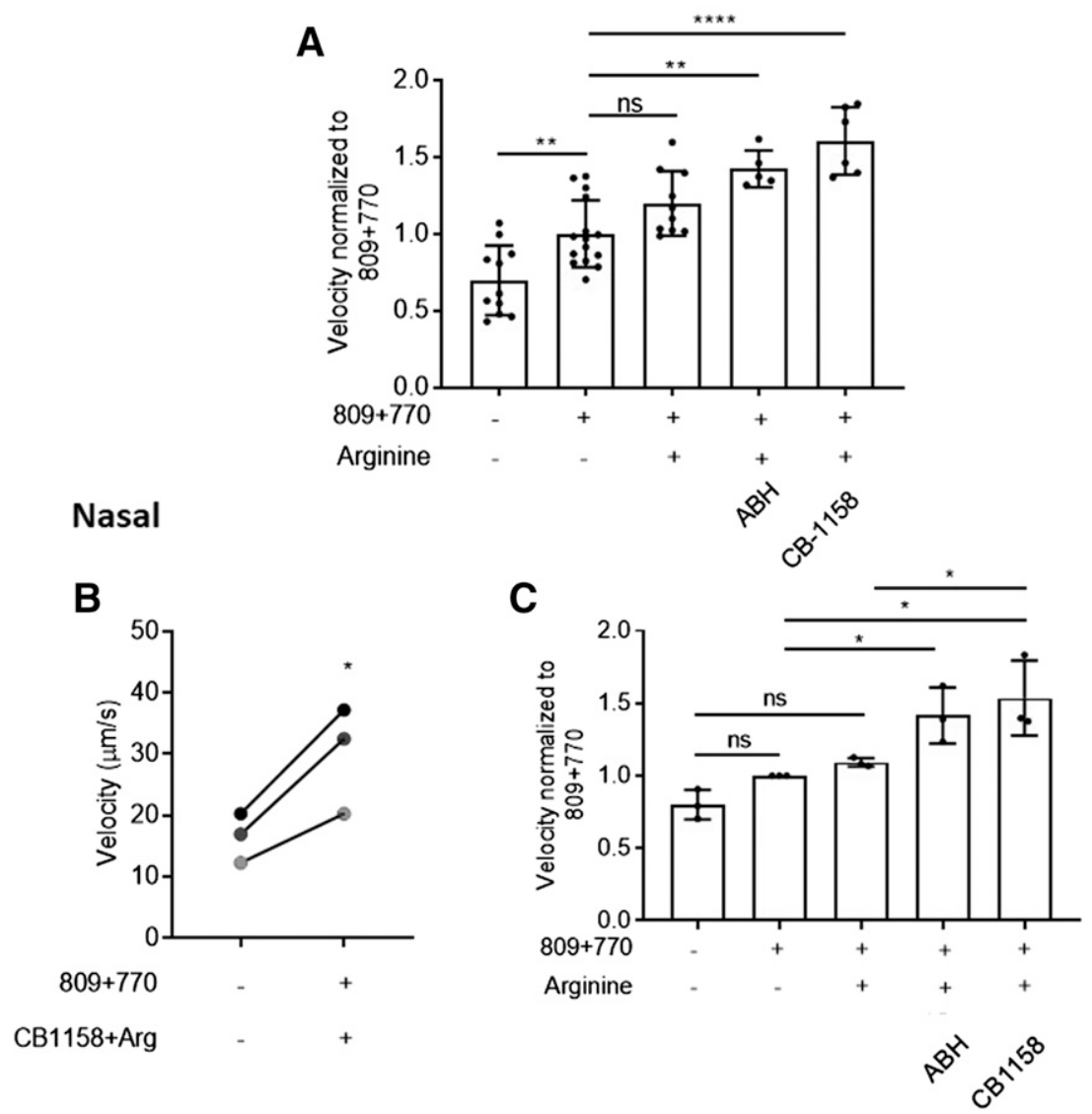

Fig. 6. Inhibition of arginase activity enhances rescue of MCCV on the surface of primary respiratory epithelial tissues by VX-809 plus VX-770. (A) Bar graph represents MCCV (micrometers per second) of F508del-CFTR primary bronchial cells with 48-hour chronic treatment of either DMSO or $3 \mu \mathrm{M}$ VX809 (mean \pm S.D.). Cells were pretreated with $1 \mathrm{mM}$ L-arginine, $10 \mu \mathrm{M}$ CB-1158, $10 \mu \mathrm{M} \mathrm{ABH}$, or buffer in the HBSS solution containing $1 \mu \mathrm{m}$ green or red polystyrene microspheres $(0.02 \%)$ following 1 hour of equilibration and 30 minutes of either $10 \mu \mathrm{M} \mathrm{FSK} \mathrm{or} 10 \mu \mathrm{M}$ FSK with $1 \mu \mathrm{M}$ VX-770 apical addition to the HBSS. WT baseline is based on the mean FSK-stimulated values of eight individual WT-CFTR primary bronchial cells. The MCCV values were normalized to each biologic replicate's respective VX-809 plus VX-770 treatment. One-way ANOVA with Tukey's multiple comparison test was performed $\left({ }^{* * P}<0.007\right.$; $* * * P<0.0001, N>4$ biologic replicates, $n=3$ technical replicates). (B) Connected line plot represents the FSK-stimulated MCCV (micrometers per second) of the fully differentiated (day 30 postseeding) F508del-CFTR primary nasal cells treated with $3 \mu$ M VX-809 following equilibration in HBSS solution containing $1 \mu \mathrm{m}$ polystyrene microspheres $(0.02 \%)$ for 1 hour. Each dot represents an individual biologic replicate $(n=2$ to 3 technical replicates). Paired $t$ test was performed ( $* P<0.05, N=3$ biologic replicates, $n=2$ technical replicates). (C) Bar graph represents MCCV of the fully differentiated (day 30 postseeding) F508del-CFTR primary nasal cells with 48 -hour chronic treatment of either DMSO or $3 \mu \mathrm{M}$ VX-809 (mean \pm S.D.). Cells were pretreated with $1 \mathrm{mM}$ L-arginine, $10 \mu \mathrm{M}$ CB-1158, $10 \mu \mathrm{M}$ ABH, or buffer in the HBSS solution containing $1 \mu \mathrm{M}$ green or red polystyrene microspheres following 1 hour of equilibration and 30 minutes of either $10 \mu \mathrm{M}$ FSK or $10 \mu \mathrm{M}$ FSK with $1 \mu \mathrm{M}$ VX-770 apical addition to the HBSS. The MCCV values were normalized to each biologic replicate's respective VX-809 plus VX-770 treatment. One-way ANOVA with Tukey's multiple comparison test was performed ( $\mathrm{ns}=$ not statistically significant, ${ }^{*} P<0.05, N=3$ biologic replicates, $n=2$ to 3 technical replicates).

(either $\mathrm{ABH}$ or $\mathrm{CB}-1158$ ) on top of corrector and potentiation modestly increased CBF.

Modulation of CBF was then measured in differentiated nasal cultures derived from three individual $\mathrm{CF}$ patients homozygous for F508del ( $n>18$ technical replicates in aggregate). Correction with VX-809 and potentiation with VX-770 increased CBF in nasal cultures by approximately $20 \%$ (Fig. 4, bottom panel). As in the case of the bronchial cultures, the addition of arginine together with arginase inhibitors augmented the CBF over that measured for nasal cultures treated with the corrector (VX-809) and the potentiator, VX-770.

MCCV on the surface of differentiated bronchial epithelial cultures was measured by tracking the velocity of fluorescent beads added to the airway surface fluid. Qualitatively, the beads on non-CF bronchial cultures moved in a sweeping hurricane-like pattern (Fig. 5A). In the case of CF cultures (homozygous for F508del), a number of bead-tracking patterns were observed, but most of the beads moved in tighter vortexlike patterns relative to the WT-CFTR bronchial cultures (Fig. 5A). The absolute velocity of such movement in WTCFTR bronchial cultures equated to approximately $40 \mu \mathrm{m} / \mathrm{s}$, 4-fold greater than CF bronchial cultures (Fig. 5B).

Bead velocity on the apical surface of $\mathrm{CF}$ bronchial cultures showed a modest increase following VX-809 plus VX-770 rescue (in cultures from more than six donors; Fig. 6A). Arginine pretreatment together with either of the two arginase inhibitors $(\mathrm{ABH}$ or $\mathrm{CB}-1158)$ further increased the bead velocity (Fig. 6A).

CF nasal cultures from three individual F508del homozygous patients (with a total of $n>18$ technical replicates) were also assessed for MCCV using the bead-tracking assay. As in the case of the bronchial cultures, preincubation with arginine and CB-1158 (10 $\mu \mathrm{M})$ enhanced the increase in 


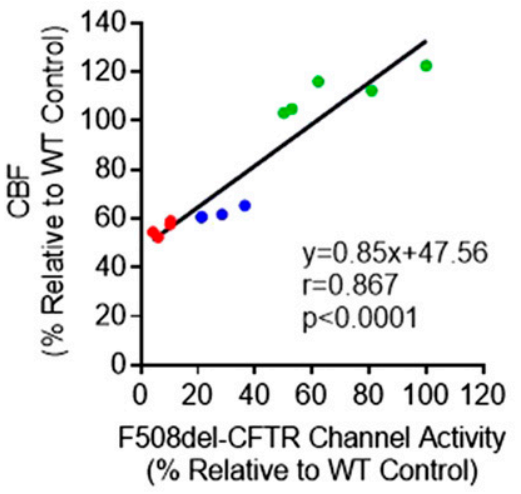

(\% Relative to WT Control)

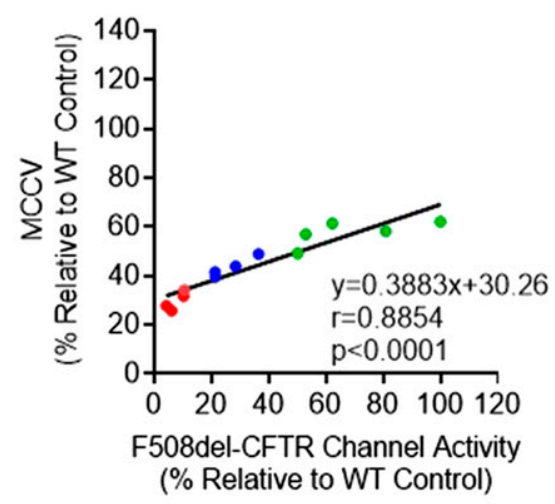

(\% Relative to WT Control)
Fig. 7. Restoration of normal channel function translates to complete rescue of CBF but only partial rescue of MCCV in VX-809 plus VX-770-treated bronchial epithelia from patients homozygous for F508del. CFTR channel activity, CBF, and MCCV outcome measurements of three conditions treated with $10 \mu$ M FSK: 48-hour DMSO (red), 48-hour VX809 plus VX-770 (blue), and 48-hour VX-809 plus VX770 with CB-1158 and arginine (green), which were normalized to experimental WT controls and plotted against channel activity. Left: CFTR channel activity was plotted against $\mathrm{CBF}$, and the equation of the line graph was generated by calculating linear regression using Spearman's correlation test $(* * * * P<0.0001$, $r=0.867)$. Right: CFTR channel activity was plotted against MCCV, and the equation of the line graph was generated by calculating linear regression using Spearman's correlation test $(r=0.8854)$.
- DMSO

-809-770

-809-770-CB1158-Arg bead velocity conferred by VX-809 plus VX-770 treatment, from 19 to $30 \mu \mathrm{m} / \mathrm{s}$ (Fig. 6B). Figure 6C shows the MCCV data from these experiments normalized relative to the VX-809 plus VX-770. This analysis shows that the augmentation of bead movement caused by arginine plus arginase inhibitors was similar in both types of cultures, bronchial and nasal.

As previously mentioned, both in vitro phenotypes, $\mathrm{CBF}$ and MCCV, are thought to reflect CFTR channel function. Hence, we predicted that there would be a positive correlation between the F508del-CFTR channel activity (measured using the FLIPR assay) and CBF or MCCV for each of the primary bronchial cultures studied. In fact, we did find a positive correlation between F508del-CFTR channel activity and CBF or MCCV when all phenotypes were normalized relative to WT-CFTR (Fig. 7).

Interestingly, the slopes of the two correlations are different, with the gain in CBF associated with increasing F508delCFTR channel function being approximately 2-fold greater than the gain in MCCV (Fig. 7). The combination of arginine, arginase inhibition, and CFTR modulators rescues both channel function and CBF to near WT levels (left panel). In contrast, complete rescue of F508del-CFTR channel activity fails to completely rescue MCCV.

\section{Discussion}

The combination of CFTR modulators, lumacaftor and ivacaftor, in ORKAMBI is modestly effective in improving lung function in some, but not all individuals bearing the common mutations, F508del-CFTR (Cholon et al., 2016). Therefore, the results of the current studies of airway epithelium from CF patients are encouraging and support the idea that the ORKAMBI effect size could be enhanced by targeting a CFTR signaling pathway, the NO pathway. In contrast to our previous studies of arginine-dependent NO activation of F508del-cftr in mouse intestine, in this study we show that, in human bronchial epithelial cells, enhancement of NO accumulation and F508del-CFTR channel activation
Fig. 8. Combinational therapy with VX-809 plus VX-770 and L-arginine-NO pathway agonists can ameliorate the CFTR channel activity, CBF, and mucociliary clearance defects observed in F508del-CFTR respiratory tissues. Increased protein kinase $\mathrm{G}(\mathrm{PKG})$ phosphorylation activates CFTR channel activity and the ATPase motor of cilia to increase ciliary beating. In CF patients, there is increased arginase expression, which results in a buildup of proline (buildup of fibrotic mass) and polyamine (inhibitor of NO synthase activity). This, in turn, decreases NO synthase expression and activity, decreases NO production, and consequently decreases CFTR channel activity and cilia motility. By using L-arginine-NO pathway agonists in VX-809 plus VX-770-rescued F508del-CFTR tissue, PKG and protein kinase A (PKA) agonists in conjunction activate corrected CFTR at the apical surface as well as the ATPase motor of cilia, resulting in a higher $\mathrm{pH}$ and less viscous environment for metachronal ciliary beating and thus mucociliary clearance to occur. 
after biosynthetic rescue in bronchial airway cells requires apical arginine, as well as a small-molecule inhibitor of arginase.

Arginase inhibition using a small molecule, CB1158, currently in clinical trial for cancer, enhanced arginine-dependent NO production and the potentiated function of the lumacaftorrescued mutant protein. This treatment also enhanced CBF and MCCV on the apical surface of the patient-derived cultures.

Interestingly, there have already been several attempts to improve lung function in $\mathrm{CF}$ patients by modulating the arginine-NO pathway through the delivery of inhaled NO (Ratjen et al., 1999), arginine supplementation (Grasemann et al., 1999, 2005a, 2013), the use of growth hormone (Grasemann et al., 2008 ) to augment NO synthase, and dietary nitrate supplementation (Kerley et al., 2016). None of these approaches led to statistically significant improvements in lung function (Grasemann et al., 1999, 2005a, 2008, 2013; Ratjen et al., 1999; Snyder et al., 2002; Taylor-Cousar et al., 2015; Kerley et al., 2016). Our in vitro studies suggest that arginine supplementation might be more effective in future clinical studies if added in combination with an inhibitor of arginase like CB1158. This suggestion is in keeping with previous studies showing that the epithelium derived from $\mathrm{CF}$ mice (Jaecklin et al., 2014) exhibits elevated arginase activity, a phenotype that would prevent intracellular arginine.

It remains to be determined whether the positive effects observed with CB1158 in combination with VX-809 plus VX770 in patient-derived airway epithelial cultures will be clinically relevant. Patient-derived primary bronchial epithelial cultures were used previously to inform CF drug development. Positive changes in F508del-CFTR channel function measured in primary airway cultures using the modulator VX-770 provided the evidence of efficacy required for their future evaluation in clinical trials (Van Goor et al., 2009; Shah, 2011; Yu et al., 2012). The correlations between effective size measured in in vitro and clinical studies are still being evaluated. The group of Sermet-Gaudelus and colleagues showed that there was a statistically significant correlation between patient-specific responses to ORKAMBI in in vitro measures of rescued F508del-CFTR channel activity in nasal cultures and improvements in lung function (Pranke et al., 2017). Similarly, the Beekman research group showed that there is also a correlation between rectal organoid swelling, an indirect measure of CFTR channel function in patient-derived rectal epithelium and that individuals' clinical improvement, measured as forced expiratory volume in 1 second (percent predicted) (Beekman, 2016; Dekkers et al., 2016). Yet, the power of in vitro assays to predict health outcomes is still being debated. For example, in contrast to the above studies, no statistically significant correlations between direct measures of CFTR channel function in patient-derived intestinal biopsies and clinical improvement after ORKAMBI were detected in studies by Mall et al. (2004) (Gräber et al., 2018).

It has been suggested that the power of in vitro assays of patient-derived tissues to predict clinical outcome will be enhanced by including multiple in vitro phenotypes from the same tissue. In the current paper, we examined three different in vitro outcomes of airway epithelial function, F508del-CFTR channel activity, CBF, and MCCV. All of these outcomes showed that there was an additive effect of arginase inhibition and CFTR modulators. These findings were not surprising because these in vitro phenotypes are thought to be related to F508del-CFTR channel modulation. As shown in the cartoon of
Fig. 8, NO accumulation is expected to directly enhance ciliary motor proteins via cyclic GMP-dependent phosphorylation in addition to its modulation of membrane-localized F508delCFTR (Wirschell et al., 2011). In fact, of the maximal CBF stimulated in CF epithelia in these studies of $9.3 \mathrm{~Hz}$, approximately $20 \%$ was inhibited by CFTRinh-172, reducing the mean CBF to $7.3 \mathrm{~Hz}$. So, it is possible that increases in CBF with CB1158 and arginine reflect stimulation of the ciliary motor and the mutant channel.

Interestingly, the slope of the correlation between changes in channel function and CBF was greater than the slope for the correlation between channel function and MCCV. The basis for this difference in slope was not studied in this work, but, in the future, we will investigate the proposal that CFTR channel modulators more easily modify the periciliary fluid than the mucus solids on top.

Each of the in vitro phenotypes, F508del-CFTR channel activity, CBF, and MCCV, were studied in both primary bronchial epithelial cultures and primary nasal epithelial cultures (Figs. and Figs. 13 -Figs. 16 ). The bronchial and nasal cultures were not derived from the same individuals, and, hence, the results are not directly comparable. But, overall, the magnitude of the responses to the interventions targeting the NO signaling pathway and the CFTR modulators, VX-809 and VX-770, is similar. These findings are consistent with those reported by Brewington et al., 2018, showing that nasal epithelial cultures serve as adequate surrogates for bronchial cultures with respect to modulator effects on F508del-CFTR channel function. Our studies of CBF plus MCCV support the claim that the effect of modulators on CF-associated phenotypes can also be modeled in nasal epithelial cultures.

In summary, we demonstrate that strategies to augment arginine-NO pathway in the context of CFTR modulation can improve CFTR channel activity and downstream airway epithelial phenotypes. Because the small-molecule inhibitor of arginase, CB1158, has been shown to be safe for treatment of cancer, we suggest that it has the potential to be repurposed as a companion therapy to be given together with ORKAMBI to patients who are homozygous for F508del-CFTR.

\section{Acknowledgments}

We recognize the exceptional service provided by Tailored Genes in generating lentivirus containing SLC6A14-GFP and stably transducing epithelial cells; Dr. Jacqueline McCormack in editing the manuscript; and consultation with Dr. Jason Chen (University of California-Irvine) regarding analysis of CBF. Primary bronchial cultures (non-CF and CF) were obtained from Dr. Philip Karp (National Institutes of Health-Iowa Culture Facility). Nasal epithelial cultures were obtained from the SickKids and CF Canada Program in Individualized Cystic Fibrosis Therapy. We also acknowledge the expertise provided by Kimberly Lau (SickKids Imaging Centre) in optimizing imaging methods for the measurement of MCCV.

\section{Authorship Contributions}

Participated in research design: Wu, Ahmadi, Wellhauser, Gonska, Moraes, Bear.

Conducted experiments: Wu, Jiang, Ahmadi, Lew, Laselva, Xia, Bartlett, Ip, Ouyang, Gonska.

Contributed new reagents or analytic tools: Wu, Jiang, Laselva, Wellhauser.

Performed data analysis: Wu, Jiang, Ahmadi, Lew, Laselva, Bartlett, Ip, Wellhauser, Gonska.

Wrote or contributed to the writing of the manuscript: Wu, Bartlett, Gonska, Moraes, Bear. 


\section{References}

Ahmadi S, Bozoky Z, Di Paola M, Xia S, Li C, Wong AP, Wellhauser L, Molinski SV, Ip W, Ouyang H, et al. (2017) Phenotypic profiling of CFTR modulators in patientderived respiratory epithelia. NPJ Genom Med 2:12.

Ahmadi S, Xia S, Wu YS, Di Paola M, Kissoon R, Luk C, Lin F, Du K, Rommens J, and Bear CE (2018) SLC6A14, an amino acid transporter, modifies the primary CF defect in fluid secretion. eLife 7:e37963.

Beekman JM (2016) Individualized medicine using intestinal responses to CFTR potentiators and correctors. Pediatr Pulmonol 51:S23-S34.

Brewington JJ, Filbrandt ET, LaRosa FJ III, Moncivaiz JD, Ostmann AJ, Strecker LM, and Clancy JP (2018) Brushed nasal epithelial cells are a surrogate for bronchial epithelial CFTR studies. JCI Insight 3 (13):e99385.

Cao H, Ouyang H, Grasemann H, Bartlett C, Du K, Duan R, Shi F, Estrada M, Seigel $\mathrm{KE}$, Coates AL, et al. (2018) Transducing airway basal cells with a helperdependent adenoviral vector for lung gene therapy. Hum Gene Ther 29:643-652

Cao H, Ouyang H, Ip W, Du K, Duan W, Avolio J, Wu J, Duan C, Yeger H, Bear CE, et al. (2015) Testing gene therapy vectors in human primary nasal epithelial cultures. Mol Ther Methods Clin Dev 2:15034.

Chen JJ, Lemieux BT, and Wong BJ (2016) A low-cost method of ciliary beat frequency measurement using iPhone and MATLAB: rabbit study. Otolaryngol Head Neck Surg 155:252-256.

Chin S, Yang D, Miles AJ, Eckford PD, Molinski S, Wallace BA, and Bear CE (2017) Attenuation of phosphorylation-dependent activation of cystic fibrosis transmembrane conductance regulator (CFTR) by disease-causing mutations at the transmission interface. J Biol Chem 292:1988-1999.

Cholon DM, Esther CR Jr, and Gentzsch M (2016) Efficacy of lumacaftor-ivacaftor for the treatment of cystic fibrosis patients homozygous for the F508del-CFTR mutation. Expert Rev Precis Med Drug Dev 1:235-243.

de Courcey F, Zholos AV, Atherton-Watson H, Williams MT, Canning P, Danahay HL, Elborn JS, and Ennis M (2012) Development of primary human nasal epithelial cell cultures for the study of cystic fibrosis pathophysiology. Am J Physiol Cell Physiol 303:C1173-C1179.

Dekkers JF, Berkers G, Kruisselbrink E, Vonk A, de Jonge HR, Janssens HM, Bronsveld I, van de Graaf EA, Nieuwenhuis EE, Houwen RH, et al. (2016) Characterizing responses to CFTR-modulating drugs using rectal organoids derived from subjects with cystic fibrosis. Sci Transl Med 8:344ra84.

Devalia JL, Sapsford RJ, Wells CW, Richman P, and Davies RJ (1990) Culture and comparison of human bronchial and nasal epithelial cells in vitro. Respir Med 84:303-312.

Eckford PD, Li C, Ramjeesingh M, and Bear CE (2012) Cystic fibrosis transmembrane conductance regulator (CFTR) potentiator VX-770 (ivacaftor) opens the defective channel gate of mutant CFTR in a phosphorylation-dependent but ATP independent manner. J Biol Chem 287:36639-36649.

Galietta LJ, Musante L, Romio L, Caruso U, Fantasia A, Gazzolo A, Romano L, Sacco O, Rossi GA, Varesio L, et al. (1998) An electrogenic amino acid transporter in the apical membrane of cultured human bronchial epithelial cells. Am J Physiol 275:L917-L923.

Gräber S, Dopfer C, Naehrlich L, Gyulumyan L, Scheuermann H, Hirtz S, Wege S, Mairbaeurl H, Dorda M, Hyde R, et al. (2018) Effects of lumacaftor-ivacaftor therapy on CFTR function in Phe508del homozygous patients with cystic fibrosis. Eur Respir J 52 (Suppl 62):PA3415.

Grasemann C, Ratjen F, Schnabel D, Reutershahn E, Vester U, and Grasemann H (2008) Effect of growth hormone therapy on nitric oxide formation in cystic fibrosis patients. Eur Respir J 31:815-821.

Grasemann H, Gärtig SS, Wiesemann HG, Teschler H, Konietzko N, and Ratjen F (1999) Effect of L-arginine infusion on airway NO in cystic fibrosis and primary ciliary dyskinesia syndrome. Eur Respir $J$ 13:114-118.

Grasemann H, Grasemann C, Kurtz F, Tietze-Schillings G, Vester U, and Ratjen F (2005a) Oral L-arginine supplementation in cystic fibrosis patients: a placebocontrolled study. Eur Respir J 25:62-68.

Grasemann H, Knauer N, Büscher R, Hübner K, Drazen JM, and Ratjen F (2000) Airway nitric oxide levels in cystic fibrosis patients are related to a polymorphism in the neuronal nitric oxide synthase gene. Am J Respir Crit Care Med 162 $2172-2176$.

Grasemann H and Pencharz PB (2013) Arginine metabolism in patients with cystic fibrosis. J Pediatr 163:317-319.

Grasemann H and Ratjen F (2012) Nitric oxide and L-arginine deficiency in cystic fibrosis. Curr Pharm Des 18:726-736.

Grasemann H, Schwiertz R, Matthiesen S, Racké K, and Ratjen F (2005b) Increased arginase activity in cystic fibrosis airways. Am $J$ Respir Crit Care Med 172: $1523-1528$.

Grasemann H, Tullis E, and Ratjen F (2013) A randomized controlled trial of inhaled L-arginine in patients with cystic fibrosis. J Cyst Fibros 12:468-474.

Jaecklin T, Duerr J, Huang H, Rafii M, Bear CE, Ratjen F, Pencharz P, Kavanagh BP, Mall MA, and Grasemann H (2014) Lung arginase expression and activity is increased in cystic fibrosis mouse models. J Appl Physiol (1985) 117:284-288.

Jih KY and Hwang TC (2013) Vx-770 potentiates CFTR function by promoting decoupling between the gating cycle and ATP hydrolysis cycle. Proc Natl Acad Sci USA 110:4404-4409.

Kerley CP, Kilbride E, Greally P, and Elnazir B (2016) Dietary nitrate acutely and markedly increased exhaled nitric oxide in a cystic fibrosis case. Clin Med Res 14 151-155.

Laselva O, Marzaro G, Vaccarin C, Lampronti I, Tamanini A, Lippi G, Gambari R Cabrini G, Bear CE, Chilin A, et al. (2018a) Molecular mechanism of action of trimethylangelicin derivatives as CFTR modulators. Front Pharmacol 9:719.

Laselva O, Molinski S, Casavola V, and Bear CE (2018b) Correctors of the major cystic fibrosis mutant interact through membrane-spanning domains. Mol Pharmacol 93:612-618.
Mall M, Kreda SM, Mengos A, Jensen TJ, Hirtz S, Seydewitz HH, Yankaskas J, Kunzelmann K, Riordan JR, and Boucher RC (2004) The DeltaF508 mutation results in loss of CFTR function and mature protein in native human colon. Gastroenterology 126:32-41.

Matsui H, Grubb BR, Tarran R, Randell SH, Gatzy JT, Davis CW, and Boucher RC (1998) Evidence for periciliary liquid layer depletion, not abnormal ion composition, in the pathogenesis of cystic fibrosis airways disease. Cell $\mathbf{9 5}$ 1005-1015.

Molinski SV, Ahmadi S, Hung M, and Bear CE (2015) Facilitating structure-function studies of CFTR modulator sites with efficiencies in mutagenesis and functional screening. J Biomol Screen 20:1204-1217.

Molinski SV, Shahani VM, Subramanian AS, MacKinnon SS, Woollard G, Laforet M, Laselva O, Morayniss LD, Bear CE, and Windemuth A (2018) Comprehensive mapping of cystic fibrosis mutations to CFTR protein identifies mutation clusters and molecular docking predicts corrector binding site. Proteins 86:833-843.

Müller L, Brighton LE, Carson JL, Fischer WA II, and Jaspers I (2013) Culturing of human nasal epithelial cells at the air liquid interface. J Vis Exp (80):e50646, doi: $10.3791 / 50646$

Ostedgaard LS, Rogers CS, Dong Q, Randak CO, Vermeer DW, Rokhlina T, Karp PH, and Welsh MJ (2007) Processing and function of CFTR-DeltaF508 are speciesdependent. Proc Natl Acad Sci USA 104:15370-15375.

Pranke IM, Hatton A, Simonin J, Jais JP, Le Pimpec-Barthes F, Carsin A, Bonnette P, Fayon M, Stremler-Le Bel N, Grenet D, et al. (2017) Correction of CFTR function in nasal epithelial cells from cystic fibrosis patients predicts improvement of respiratory function by CFTR modulators. Sci Rep 7:7375.

Ratjen F, Gärtig S, Wiesemann HG, and Grasemann H (1999) Effect of inhaled nitric oxide on pulmonary function in cystic fibrosis. Respir Med 93:579-583.

Ratjen F, Hug C, Marigowda G, Tian S, Huang X, Stanojevic S, Milla CE, Robinson PD, Waltz D, Davies JC, et al. (2017) Efficacy and safety of lumacaftor and ivacaftor in patients aged 6-11 years with cystic fibrosis homozygous for F508delCFTR: a randomised, placebo-controlled phase 3 trial. Lancet Respir Med $\mathbf{5}$ $557-567$.

Shah S (2011) VX-770, a CFTR potentiator, may have a potential clinical benefit in a subgroup of people with cystic fibrosis. Thorax 66:984.

Snyder AH, McPherson ME, Hunt JF, Johnson M, Stamler JS, and Gaston B (2002) Acute effects of aerosolized S-nitrosoglutathione in cystic fibrosis. Am J Respir Crit Care Med 165:922-926.

Steggerda SM, Bennett MK, Chen J, Emberley E, Huang T, Janes JR, Li W, MacKinnon AL, Makkouk A, Marguier G, et al. (2017) Inhibition of arginase by CB1158 blocks myeloid cell-mediated immune suppression in the tumor microenvironment. J Immunother Cancer 5:101.

Sutto Z, Conner GE, and Salathe M (2004) Regulation of human airway ciliary beat frequency by intracellular $\mathrm{pH}$. J Physiol 560:519-532.

Taylor-Cousar JL, Wiley C, Felton LA, St Clair C, Jones M, Curran-Everett D, Poch K, Nichols DP, Solomon GM, Saavedra MT, et al. (2015) Pharmacokinetics and tolerability of oral sildenafil in adults with cystic fibrosis lung disease. $J$ Cyst Fibros 14:228-236.

Treharne KJ, Crawford RM, Xu Z, Chen JH, Best OG, Schulte EA, Gruenert DC, Wilson SM, Sheppard DN, Kunzelmann K, et al. (2008) Protein kinase CK2, cystic fibrosis transmembrane conductance regulator, and the deltaF508 mutation: F508 deletion disrupts a kinase-binding site. J Biol Chem 283:25103.

van den Berg MPM, Kurhade SH, Lunev S, van Faassen HJR, Bos IST, Zuidhof AB Groves M, Kema IP, Dömling A, Meurs H, et al. (2018) The in vitro and ex vivo efficacy of novel arginase inhibitors for the treatment of allergic asthma. Am $J$ Respir Crit Care Med 197:A5806.

Van Goor F, Hadida S, Grootenhuis PD, Burton B, Cao D, Neuberger T, Turnbull A Singh A, Joubran J, Hazlewood A, et al. (2009) Rescue of CF airway epithelial cell function in vitro by a CFTR potentiator, VX-770. Proc Natl Acad Sci USA 106 18825-18830.

Van Goor F, Hadida S, Grootenhuis PD, Burton B, Stack JH, Straley KS, Decker CJ, Miller M, McCartney J, Olson ER, et al. (2011) Correction of the F508del-CFTR protein processing defect in vitro by the investigational drug VX-809. Proc Natl Acad Sci USA 108:18843-18848.

Van Goor F, Yu H, Burton B, and Hoffman BJ (2014) Effect of ivacaftor on CFTR forms with missense mutations associated with defects in protein processing or function. J Cyst Fibros 13:29-36.

Wainwright CE, Elborn JS, Ramsey BW, Marigowda G, Huang X, Cipolli M, Colombo C, Davies JC, De Boeck K, Flume PA, et al.; TRAFFIC Study Group TRANSPORT Study Group (2015) Lumacaftor-ivacaftor in patients with cystic fibrosis homozygous for Phe508del CFTR. N Engl J Med 373:220-231.

Wirschell M, Yamamoto R, Alford L, Gokhale A, Gaillard A, and Sale WS (2011) Regulation of ciliary motility: conserved protein kinases and phosphatases are targeted and anchored in the ciliary axoneme. Arch Biochem Biophys 510:93-100.

Yu H, Burton B, Huang CJ, Worley J, Cao D, Johnson JP Jr, Urrutia A, Joubran J, Seepersaud S, Sussky K, et al. (2012) Ivacaftor potentiation of multiple CFTR channels with gating mutations. J Cyst Fibros 11:237-245.

Zhang W, Zhang X, Zhang YH, Strokes DC, and Naren AP (2016) Lumacaftor/ivacaftor combination for cystic fibrosis patients homozygous for Phe508del-CFTR. Drugs Today (Barc) 52:229-237.

Address correspondence to: Dr. Christine E. Bear, Peter Gilgan Centre for Research and Learning, Hospital for Sick Children, 686 Bay Street, Toronto, Canada. E-mail: bear@sickkids.ca 